\title{
Catalytic Activity in Oxidation Reactions of Anionic Iron(III) Porphyrins Immobilized on Raw and Grafted Chrysotile
}

\author{
Shirley Nakagaki, * Kelly A. D. F. Castro, Guilherme S. Machado, Matilte Halma, \\ Sueli M. Drechsel and Fernando Wypych
}

Departamento de Química, Universidade Federal do Paraná, CP 19081, Curitiba-PR, Brazil

\begin{abstract}
Crisotila natural e quimicamente modificada foi usada como matrizes para imobilização de ferroporfirinas de segunda geração. As atividades catalíticas dos sólidos obtidos foram avaliadas em reações de oxidação de cicloexano, usando iodosilbenzeno como oxidante. Os resultados de catálise foram analisados e comparados aos obtidos pela imobilização das mesmas ferroporfirinas em sílica funcionalizada com 3-APTS, derivadas do tratamento ácido da crisotila. Os resultados preliminares mostraram que os catalisadores são eficientes e seletivos para a obtenção de álcool.
\end{abstract}

Natural and grafted chrysotile were used as matrices for the immobilization of second generation iron porphyrins. The catalysts obtained were evaluated in the oxidation reaction of cyclohexane, using iodosylbenzene as oxidant agent. The catalyst activity for different conditions was compared with results for the same porphyrins immobilized with 3-APTS grafted disordered silica, obtained from acid-leached chrysotile. Preliminary results have shown that high activity can be obtained with short reaction times, and that the reaction is specific for alcohol.

Keywords: iron porphyrin, supported catalysts, chrysotile, grafting, oxidation, catalysis

\section{Introduction}

Metalloporphyrins are important examples of macrocyclic complexes and have attracted much interest in the study of various oxygenation reactions of hydrocarbons under mild conditions. ${ }^{1-4}$ This class of compounds is used in solution or following immobilization in organic amorphous polymers and crystalline inorganic materials such as silica, ${ }^{5-7}$ zeolites,${ }^{8,9}$ clay from the smectite group (montmorillonite) $)^{10,11}$ and others. ${ }^{12-16}$ The use of metalloporphyrins substituted with electron-withdrawing groups (the so-called second generation porphyrins ${ }^{17}$ ) and their immobilization, has resulted in efficient and selective catalysts for oxidation reactions, since the support can impose shape selectivity and promote a special environment, favoring the approach of the substrate to the active catalytic specie..$^{5-7,12,13,18}$ In addition, the immobilization may prevent molecular aggregation or bimolecular self-destruction reactions, which can lead to deactivation of catalytically active metalloporphyrin species. The immobilization of metalloporphyrins is also associated with an easily recyclable solid, which can be

*e-mail: shirley@quimica.ufpr.br reused. ${ }^{19}$ To obtain better catalytic systems, new and alternative matrices for immobilization should be studied. Nanotubes of chrysotile are an interesting option, because of their highly hydroxilated surface and large surface area.

Chrysotile, an alternative source of highly hydroxylated silica, is classified in the kaolin/serpentine 1:1 layer mineral group and is characterized by tri-octahedral site occupancy. ${ }^{20-22}$ Chrysotile is the magnesium-based mineral analogous to kaolinite, and consists of sheets of brucitelike $\mathrm{Mg}(\mathrm{OH})_{2}$ structure linked to sheets of silica, producing layers of the 1:1 group minerals. ${ }^{20}$ The mismatch of the brucite-like octahedral sheet with the silica tetrahedral sheet causes the curvature of the layers, which can roll into tight tubes, the characteristic chrysotile fibers. ${ }^{21} \mathrm{~A}$ drastic leaching of chrysotile fibers by concentrated acid transforms this natural polymer by removing the brucite-like layer, resulting in an excellent source of hydrated disordered silica ${ }^{23,24}$ with physico-chemical characteristics similar to silica gel.

The functionalization of layered mineral supports (grafting) is a frequently employed, useful strategy for preparing the inorganic support for the immobilization of catalyst molecules such as metallocomplexes. ${ }^{25,26}$ Grafting reactions can occur by establishing covalent bonds 
between the reactive groups of the layer and an adequate reactant molecule, which ensures higher chemical, structural and thermal stability for the compound. These reactions can be restricted to the crystal surface (the basal spacing remains unchanged) or layer surface (in this case an interlayer expansion occurs, if the single layers are restacked). These compounds can be collectively defined as "hybrid" materials, or more specifically, "surfacemodified inorganic layered materials".

In this paper, we report the immobilization of the anionic iron ${ }^{\text {III }}$ porphyrins $\left\{\mathrm{Na}_{4}[\mathrm{Fe}(\mathrm{TDFSPP})]\right\} \mathrm{Cl}$ and $\left\{\mathrm{Na}_{4}[\mathrm{Fe}\right.$ (TCFSPP)] $\} \mathrm{Cl}$ (Figure 1) in raw and silanized chrysotile and its use as catalysts for oxidation reactions. Although chrysotile has a neutral structure and belongs to the same category as the clay minerals, no report has been found so far of its use as support for the immobilization of molecules with catalytic activity.

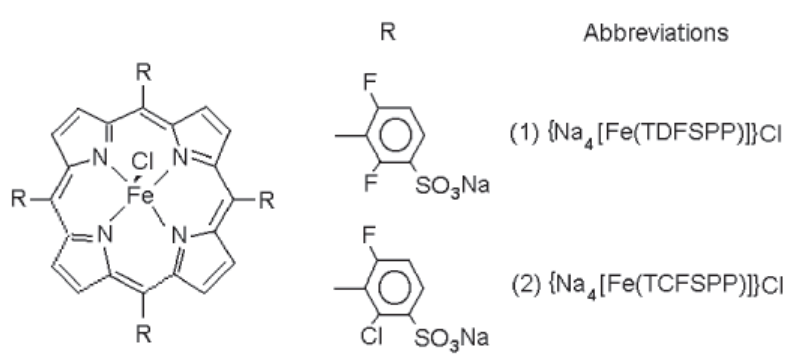

Figure 1. Schematic representation of iron(III) porphyrins: $\left\{\mathrm{Na}_{4}[\mathrm{Fe}(\mathrm{TDFSPP})]\right\}^{+}$ion $[5,10,15,20$-tetrakis(2,6-difluor-3-sulfonatophenyl)porphyrinato] iron(III) sodium salt and $\left\{\mathrm{Na}_{4}[\mathrm{Fe}(\mathrm{TCFSPP})]\right\}^{+}$; ion [5,10,15,20-tetrakis(2-chloro-6-fluor-3-sulfonatophenyl)porphyrinato] iron(III) sodium salt.

\section{Experimental}

\section{Materials}

All chemicals used in this study were purchased from Aldrich, Sigma or Merck and were of analytical grade. The pale gray chrysotile sample (Chrys) with a fiber length less than $2.0 \mathrm{~mm}$ (SAMA7ML) was supplied by SAMA - Mineração de Amianto Ltda, mined in Uruaçu, state of Goiás, Brazil. The white disordered silica solid was obtained by treating chrysotile with hydrochloric acid, as previously described. ${ }^{21,23}$ Iodosylbenzene (PhIO) was synthesized by hydrolysis of iodosylbenzenediacetate. ${ }^{26}$ The solid was carefully dried under reduced pressure and kept at $5{ }^{\circ} \mathrm{C}$. The purity was periodically controlled by iodometric titration. $^{27}$ (3-aminopropyl)triethoxysilane (3-APTS), $\mathrm{NH}_{2}\left(\mathrm{CH}_{2}\right)_{3} \mathrm{Si}\left(\mathrm{CH}_{2} \mathrm{CH}_{2} \mathrm{O}\right)_{3}$ (Aldrich), toluene (Synth) and ethanol (Nuclear) were all reagent grade. Deionized water was used in all experimental procedures.
Porphyrins. Free base porphyrins $\mathrm{Na}_{4}\left[\mathrm{H}_{2}\right.$ (TDFSPP) $]$ and $\mathrm{Na}_{4}\left[\mathrm{H}_{2}\right.$ (TCFSPP) $]$ were synthesized, purified and characterized following the methodology previously described. ${ }^{28-31}$

Iron(III) porphyrins. Iron(III) porphyrins (FePor) were obtained by metallation of the free ligand with ferrous chloride tetrahydrate in DMF following the method described by Adler and co-workers. ${ }^{32,33}$ Purification of the iron porphyrins was performed by column chromatography filled with Sephadex, using deionized water as eluent. The products were characterized by UV-Visible and EPR spectrometry and the data were consistent with the expected compound after the metallation reaction. $\mathbf{A}$ : [Fe(TDFSPP)Cl] $]^{4-}$ (deionized water): $390 \mathrm{~nm}\left(\varepsilon=37 \times 10^{3}\right.$ $\left.\mathrm{L} \mathrm{mol}^{-1} \mathrm{~cm}^{-1}\right), 504 \mathrm{~nm}\left(\varepsilon=3 \times 10^{3} \mathrm{~L} \mathrm{~mol}^{-1} \mathrm{~cm}^{-1}\right)$. B: $[\mathrm{Fe}(\mathrm{TCFSPP}) \mathrm{Cl}]^{4-}$ (deionized water) $390 \mathrm{~nm}\left(\varepsilon=15 \times 10^{3}\right.$ $\mathrm{L} \mathrm{mol}^{-1} \mathrm{~cm}^{-1}$ ). The negative charges and the chloride counter ion will be omitted in the text for simplification purposes.

\section{Immobilization of ironporphyrins}

For the immobilization of the iron porphyrins A and B in 3-APTS grafted chrysotile (FePor/Chrys-APTS), the solid was suspended in $20 \mathrm{~mL}$ of deionized water under magnetic stirring. Drops of $5 \% \mathrm{HCl}$ aqueous solution were added until $\mathrm{pH} 5$ and the suspension was magnetically stirred for approximately 15 minutes. After that, a solution of the FePor $\left(10^{-2} \mathrm{mmol}\right.$ of iron(III) porphyrin (A or B) in $5 \mathrm{~mL}$ of deionized water) was added to the container holding the suspension. After finishing the addition of the iron(III) porphyrin solution, a reflux system was adapted and the mixture was kept at a temperature of approximately $100{ }^{\circ} \mathrm{C}$ for 5 hours. For the immobilization in raw chrysotile (FePor/ Chrys) the experiments were performed in the same way, except without acidification of the suspension.

After $5 \mathrm{~h}$ of reaction, all the solids were filtered and washed with deionized water and the supernatant was stored and analyzed by UV-Vis spectroscopy with the objective of quantifying any iron(III) porphyrin that might have been removed from the matrix. The light-green solids obtained were dried in a laboratory oven at $70{ }^{\circ} \mathrm{C}$. In all the experiments, the immobilization was of $100 \%$, relative to the initial amount of iron(III) porphyrin in the solution (iron porphyrin/solid loading: FePor/Chrys $=2.4 \times 10^{-5}$ iron porphyrin $\mathrm{mol} / \mathrm{g}$ solid and FePor/Chrys-APTS $=2.6$ $\times 10^{-5}$ iron porphyrin $\mathrm{mol} / \mathrm{g}$ solid).

\section{Catalytic oxidation reactions}

Catalytic oxidation reactions were carried out in a 2 $\mathrm{mL}$ thermostatic glass reactor equipped with a magnetic 
stirrer inside of a dark chamber. In a standard experiment within the reactor, solid catalyst and iodosylbenzene (FePor:PhIO molar ratio: 1:50) were suspended in 0.380 $\mathrm{mL}$ of solvent (dichloromethane-acetonitrile 1:1 mixture $\mathrm{v} / \mathrm{v}$ ) and degassed with argon during $10 \mathrm{~min}$ inside a 2 $\mathrm{mL}$ vial. The substrate (cyclohexane, FePor:substrate molar ratio $=1: 5000$ ) was added and the oxidation reaction was carried out during 1 to 24 hours, under magnetic stirring. To eliminate the excess of iodosylbenzene, sodium sulfite was added and the products of the reaction were separated from the solid catalyst by exhaustive washing and centrifugation of the solid with an acetonitriledichloromethane mixture. The extracted solution was analyzed by capillary Gas Chromatography and the amount of the products was determined by using the internal standard method.

\section{Techniques used}

For the X-ray diffraction measurements, self-oriented films were placed on neutral glass sample holders. As the films are prepared by simple deposition in the glass sample holder the thickness cannot be high because this will interfere in the geometry of the measurement. The measurements were performed in reflection mode using a Shimadzu XRD-6000 diffractometer operating at $40 \mathrm{kV}$ and $40 \mathrm{~mA}(\mathrm{Cu}-\mathrm{K} \alpha$ radiation $\lambda=1.5418 \AA)$ with a dwell time of $1^{\circ} \mathrm{min}^{-1}$.

FTIR spectra (Fourier Transform Infrared Spectroscopy) were recorded on a Biorad 3500 GX spectrophotometer in the range of 400 to $4000 \mathrm{~cm}^{-1}$, using $\mathrm{KBr}$ pellets. $\mathrm{KBr}$ was crushed with a small amount of the solids and the spectra were collected with a resolution of $4 \mathrm{~cm}^{-1}$ and accumulation of 32 scans.

UV-Vis spectra (Ultra-violet and Visible spectroscopy) were recorded in the $200-800 \mathrm{~nm}$ range in an HP 8452A Diode Array Spectrophotometer.

Transmission electron microscopy (TEM) analyses were done using a JEOL 1200EX-II microscope, operating at $110 \mathrm{KV}$. Fine powder of the sample was carefully dispersed over a diffraction grating to perform the brightfield analyses.

Products from catalytic oxidation reactions were identified using a Shimadzu CG-14B gas chromatograph (flame ionization detector) with a DB-WAX capillary column (J \& W Scientific).

\section{Results and Discussion}

Figure 2 shows the FTIR spectra of raw chrysotile (Figure 2a), chrysotile grafted with 3-APTS (Chrys-APTS)
(Figure $2 \mathrm{~b}$ ), and the solids obtained by the immobilization of iron porphyrins (Figure 2c-2f). For comparison purposes, the infrared spectra of both iron porphyrins are also presented: Fe(TDFSPP) (Figure 2g) and Fe(TCFSPP) (Figure 2h).

The main characteristic of the spectra of raw chrysotile (Figure 2a) is the presence of the band at 3686 and $3644 \mathrm{~cm}^{-1}$, attributed to the surface hydroxide groups of the brucite-like sheet. ${ }^{34,35}$ The bands at 3451 and 1630 $\mathrm{cm}^{-1}$ were attributed to water molecules adsorbed to the chrysotile tubes. The bands at 1076, 1024 and $955 \mathrm{~cm}^{-1}$ were attributed to symmetric stretching vibrations of $\mathrm{Si}$ $\mathrm{O}-\mathrm{Si}$ and Si-O bonds. ${ }^{36}$ The band at $610 \mathrm{~cm}^{-1}$ is attributed to internal vibration of the $\mathrm{Mg}-\mathrm{O}$ bond.

The corresponding spectra for Chrys-APTS (Figure 2b) presents a lower intensity band at $2928 \mathrm{~cm}^{-1}$, for typical $\mathrm{C}-\mathrm{H}$ stretching vibration, attributed to the organic moiety bonded to the surface of chrysotile. Given the low intensity of the bands, we assume the presence of a small amount of grafted 3-APTS. The FTIR spectra of the solids Fe(TCFSPP)/Chrys (Figure 2c), Fe(TCFSPP)/Chrys-APTS (Figure 2d), Fe(TDFSPP)/Chrys (Figure 2e) and Fe(TDFSPP)/Chrys-APTS (Figure 2f), presents the characteristic bands of raw chrysotile. The bands of iron porphyrins and the grafted 3-APTS are not visible because the intensity of bands of the mineral is much higher. It is

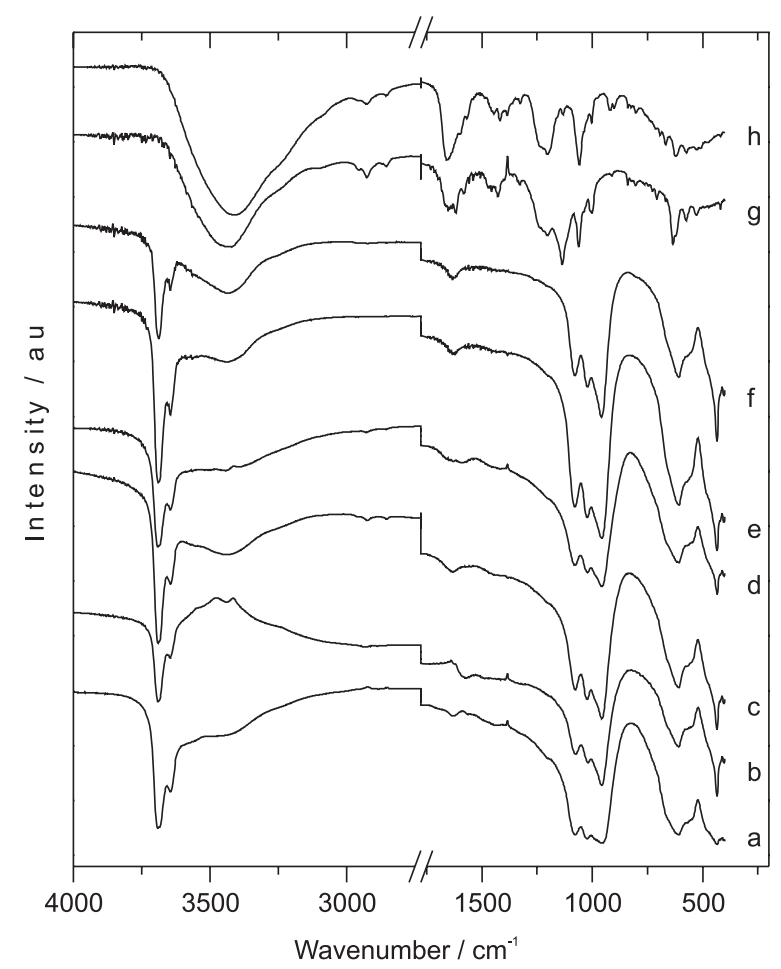

Figure 2. FTIR spectra of raw chrysotile (a), Chrys-APTS (b), Fe(TCFSPP)/Chrys (c), Fe(TCFSPP)/Chrys-APTS (d), Fe(TDFSPP)/Chrys (e) and Fe(TDFSPP)/Chrys-APTS (f) and the iron porphyrins Fe(TDFSPP) (g) $\mathrm{Fe}(\mathrm{TCFSPP})(\mathrm{h})$. 
also supposed that the grafted 3-APTS molecules could be submitted to a partial hydrolysis, as all the reactions were performed in aqueous acidic media.

Figure 3 shows the powder X-ray diffraction patterns of raw chrysotile (Figure 3a), Chrys-APTS (Figure 3b), Fe(TCFSPP)/Chrys (Figure 3c), Fe(TCFSPP)/Chrys-APTS (Figure 3d), Fe(TDFSPP)/Chrys (Figure 3e) and Fe(TDFSPP)/Chrys-APTS (Figure 3f). As would be expected for surface grafting and immobilization, all the samples present the X-ray diffraction pattern of raw chrysotile with a typical basal distance of $7.36 \AA$, characteristic of raw chrysotile. ${ }^{34,35}$ As described previously,$^{34}$ this sample has a characteristic diffraction peak positioned at $9.5^{\circ}(2 \theta)(9.31 \AA)$, attributed to a small contamination with talc.

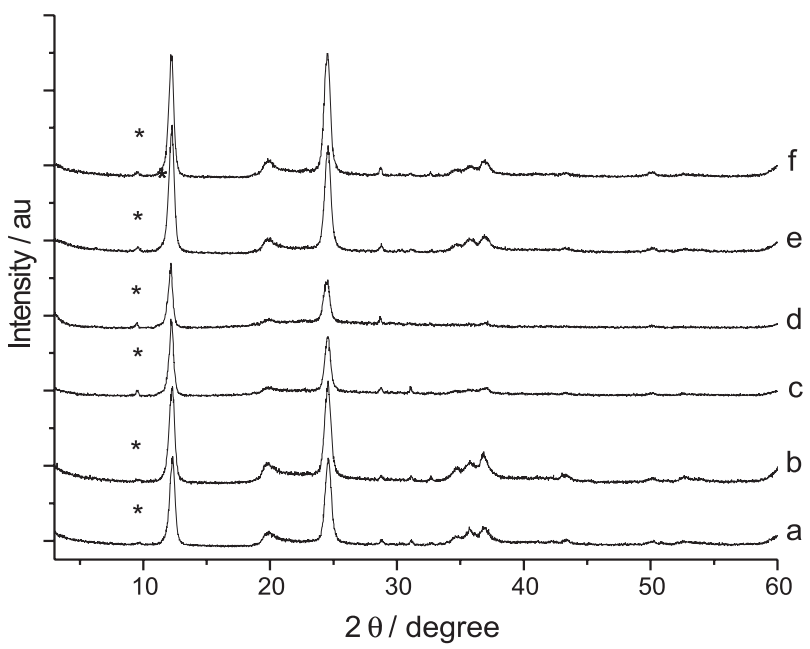

Figure 3. Powder X-ray diffraction patterns of raw chrysotile (a), ChrysAPTS (b), Fe(TCFSPP)/Chrys (c), Fe(TCFSPP)/Chrys-APTS (d), $\mathrm{Fe}(\mathrm{TDFSPP}) /$ Chrys (e) and Fe(TDFSPP)/Chrys-APTS (f). * = Talc.

Although the FTIR and X-ray diffraction techniques do not attest the presence of the iron porphyrin in the solids, the presence of the iron(III) porphyrins in the solid FePor/Chrys and FePor/Chrys-APTS was confirmed by UV-Vis spectra of solid, in Nujol mineral oil (Figure 4). The measurements suggest that no demetallation (characterized by a blue-shift of the Soret band that is associated with a significant amount of free base porphyrin ${ }^{12-14}$ ) nor significant exchange of the $\mathrm{Fe}^{\mathrm{III}}$ ion with the supports occurred during the preparation process. The Soret peaks at $417 \mathrm{~nm}$ for Fe(TCFSPP)/Chrys (Figure 4c) and $\mathrm{Fe}$ (TCFSPP)/Chrys-APTS (Figure 4d) and at $411 \mathrm{~nm}$ for Fe(TDFSPP)/Chrys (Figure 4e) and Fe(TDFSPP)/ Chrys-APTS (Figure 4f) were red-shifted when compared to those of iron porphyrins in solution (methanol:water), $388 \mathrm{~nm}$ for Fe(TCFSPP) and $392 \mathrm{~nm}$ for Fe(TDFSPP). A similar behavior was observed previously, ${ }^{19}$ when metalloporphyrins were immobilized in different inorganic supports. ${ }^{10,12-14,36}$ This behavior was attributed to steric constraints caused by the support, which modified the iron porphyrin structure substantially in these supported catalysts. ${ }^{33}$ Figure $4 \mathrm{a}$ and Figure $4 \mathrm{~b}$ show the spectra of raw chrysotile and chrysotile-APTS, where no peaks are present.

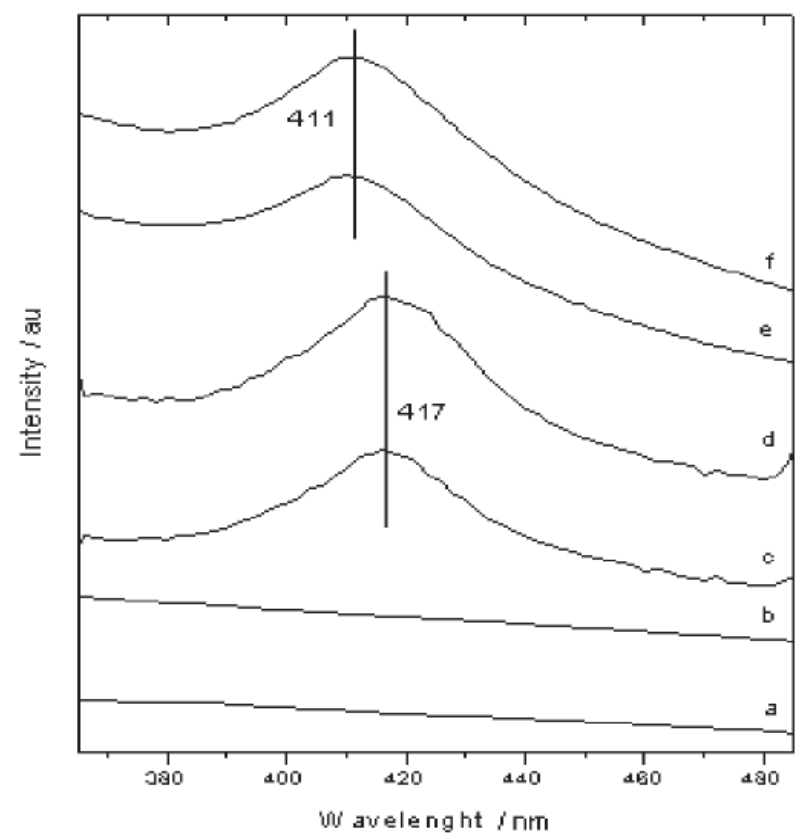

Figure 4. UV-Vis spectra of raw solid chrysotile (a), chrysotile-APTS (b), Fe(TCFSPP)/Chrys (c), Fe(TCFSPP)/Chrys-APTS (d), Fe(TDFSPP)/ Chrys (e) and Fe(TDFSPP)/Chrys-APTS (f).

The TEM measurements of Fe(TCFSPP) immobilized in 3-APTS grafted chrysotile (Figure $5 \mathrm{a}, \mathrm{c}$ ) and raw chrysotile (Figure $5 \mathrm{~b}, \mathrm{~d}$ ) have shown that the fibers did not experiment major changes in the morphology of the single fibers. In high resolution images (Figure $5 \mathrm{c}$,d), a corrugation of the surface was observed, attributed to the grafting and immobilization of the iron porphyrin at the surface of the fibers, with a single layer coverage. For the other samples (Fe(TDFSPP)/Chrys-APTS and $\mathrm{Fe}$ (TDFSPP)/Chrys) similar results were obtained (not shown).

The catalytic activities of both iron porphyrin supported catalysts (heterogeneous catalysis) and in solution (homogeneous catalysis) were investigated on the oxidation of weakly reactive alkanes such as cyclohexane. It is a very useful substrate and frequently used for the $\mathrm{Fe}^{\mathrm{IIII}}$ porphyrin-catalyzed hydroxylation by iodosylbenzene $(\mathrm{PhIO})^{6,10,37,38}$ and was used as test reaction to compare the catalyst activity of the iron porphyrins. The results are presented in Table 1 in comparison with the same porphyrins immobilized in 3-APTS grafted disordered silica, obtained from acid leached chrysotile. ${ }^{23}$ 

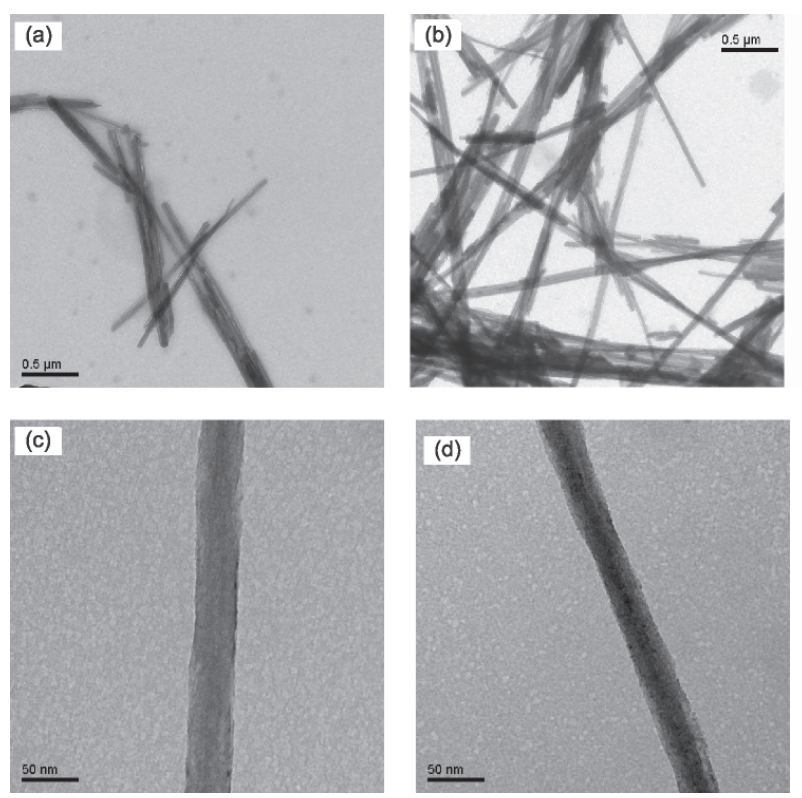

Figure 5. TEM bright-field image obtained from the samples Fe(TCFSPP) Chrys-APTS (a,c) and Fe(TCFSPP)/Chrys (b,d).

When using 1.1 to $1.4 \mathrm{mmol}$ of substrate in the solvent mixture $\mathrm{CH}_{3} \mathrm{CN}: \mathrm{CH}_{2} \mathrm{Cl}_{2}(1: 1 \mathrm{v} / \mathrm{v}$ ratio), the supported catalysts do not release their iron porphyrin, and different proportions of $\mathrm{PhIO}$ relative to the iron porphyrin supported catalyst led to selective formation of alcohol (based on PhIO) within 1 and 24h. The consumption of $\mathrm{PhIO}$ in all reactions was monitored by the presence of iodobenzene (PhI).

The low solubility of the iron porphyrins in a $\mathrm{CH}_{2} \mathrm{Cl}_{2}: \mathrm{CH}_{3} \mathrm{CN}(1: 1 \mathrm{v} / \mathrm{v}$ ratio) solvent mixture in homogeneous catalysis certainly is an important factor responsible for the low yields (and low turnover number $\mathrm{TN}$ ) of the iron porphyrin itself. The possibility of the molecular interaction of the Fe(TDFSPP) species in solution could be another reason for the low catalytic activity observed in the homogeneous catalysis because it can be accompanied by $\mu$-oxo complex formation..$^{17,37-39}$ The oxidative degradation of the catalyst in solution is frequently responsible for the low yield in catalytic reactions using metalloporphyrins ${ }^{17}$ but not for second generation iron porphyrins and in cases with a low FePor/oxidant molar ratio such as was used in run 1 (1:50).

For the iron porphyrin Fe(TDFSPP) immobilized in the three different supports in the optimum condition (24h), $61 \%$ of alcohol yield was observed when the inorganic support used was Si-APTS (run 3). When the three inorganic supports were compared and using the same porphyrin (Fe(TDFSPP)), it is clearly seen that the best performance is obtained for FePor/Si-APTS, compared with the same porphyrin immobilized in raw chrysotile (run 5) and grafted chrysotile (run 7) (for $24 \mathrm{~h}$ of reaction). This behavior can be explained by the higher surface for the silica obtained from acid leached chrysotile, leading to a better contact of the grafted active site with the oxygen donor (PhIO) and the substrate. Although the surface areas of the supports are not available, the increase of the surface area after the acid is explained by the opening and flattening of the tubes into the form of nanobelts. ${ }^{34}$ These nanobelts of disordered silica are produced from concentric tubes of chrysotile, exposing surfaces initially within the tubes and therefore not available for the grafting reactions of raw chrysotile. Another important consequence of the opening of the tubes

Table 1. Hydroxylation of cyclohexane by PhIO catalyzed by iron(III) porphyrins, Fe(TDFSPP) and Fe(TCFSPP), in homogeneous media and by the supported iron(III)porphyrins in heterogeneous media

\begin{tabular}{lcccc}
\hline Catalyst & Run & time / (h) & Alcohol yield / (\%) & TN \\
\hline Fe(TDFSPP) & 1 & 1 & 13 & 9 \\
Fe(TDFSPP)/Si-APTS ${ }^{23}$ & 2 & 1 & 36 & 19 \\
Fe(TDFSPP)/Chrys & 3 & 24 & 12 & 34 \\
Fe(TDFSPP)/Chrys-APTS & 4 & 1 & 35 & 7 \\
Fe(TCFSPP) & 5 & 24 & 16 & 9 \\
Fe(TCFSPP)/Si-APTS & 6 & 23 & 25 & 15 \\
& 7 & 1 & 48 & 3 \\
Fe(TCFSPP)/Chrys & 8 & 1 & 33 & 8 \\
& 9 & 24 & 49 & 29 \\
Fe(TCFSPP)/Chrys-APTS & 10 & 1 & 44 & 23 \\
& 11 & 1 & 60 & 30 \\
\hline
\end{tabular}

Iron porphyrin:iodosylbenzene:cyclohexane molar ratio = 1:50:5000. The hydroxylation reaction selectivity (\% alcohol:\% ketone) was higher than 10 for all reactions and conditions. Control reactions carried out using Si-APTS (silica derivates from raw chrysotile) or Chrys (raw Chrysotile) or Chrys-APTS (pure 3-APTS grafted Chrysotile) as catalyst show alcohol yields below $2 \% . \mathrm{TN}=$ mol products per mol catalyst. The yields were based on the iodosylbenzene used. The standard error is less than $5 \%$. 
is that both sides of the silica nanobelts become available to the grafting reaction, which is otherwise limited to the outer surface area of the raw chrysotile nanotubes.

Considering the same amount of matrix and a fixed similar amount of iron porphyrin distributed on the surface of the matrix (2.0 to $2.6 \times 10^{-2} \mathrm{mmol}$ of iron porphyrin per $\mathrm{g}$ of solid), in the case of acid-leached chrysotile the active sites will be better dispersed and consequently more available to the access of the reagents.

The grafted 3-APTS molecule seems to contribute negatively to the active site (run 7) in comparison with immobilization in raw chrysotile (run 5), possibly because the bonding of the $\mathrm{NH}_{2}$ surface groups interacting with the iron porphyrin reduces the activity of the catalytic site.

In the case of $\mathrm{Fe}$ (TCFSPP) the effect is not so pronounced: the catalytic activities in 24 hours are always similar and independent of surface area. This behavior can perhaps be related to the polarity of the solvent used during the catalytic experiments. For the Fe(TCFSPP) iron porphyrin, the best yield for the immobilized system was obtained in run $13(60 \%$ alcohol). In general, the Fe(TDFSPP) catalyst and its Fe(TCFSPP) counterpart display opposite behavior regarding the following: Fe(TCFSPP) presents a similar or substantially better yield in homogeneous catalysis (run 8) when compared with the case where this iron porphyrin is immobilized.

In homogeneous catalysis, one ortho-chlorine substituent in each meso-phenyl porphyrin groups in the Fe(TCFSPP) can avoid molecular interactions, which can deactivate (by destruction of the iron porphyrin or dimerization) and/or avoid the catalytic active specie formation. ${ }^{8,24}$ Therefore, higher yields in oxidation reactions are expected for this iron porphyrin in comparison with $\mathrm{Fe}$ (TDFSPP). In contrast, after immobilization, the better catalytic results observed for Fe(TDFSPP) could be due to an easy access of PhIO and substrate to the iron site, based on the small size of the two ortho-fluorine substituents in comparison with orthochlorine substituents from Fe(TCFSPP). ${ }^{24}$ Besides, the immobilization process avoids any molecular interaction possible in homogeneous catalysis, mainly when a low percentage of iron porphyrin immobilized in the Si-3APTS solid is used.

In summary the preliminary catalytic results of cyclohexane oxidation showed that the catalyst performance both iron porphyrins changes upon varying the reaction time from 1 to $24 \mathrm{~h}$. The catalytic results are dependent on the particular characteristics of each porphyrin structure and of the support. The most influential factor is probably their surface area. The use of other substrates (linear alkanes) is currently under way, as is the investigation of the kinetic behavior of the catalyst.

\section{Conclusions}

The supports before and after grafting reactions and immobilization showed the same profile when analyzed through X-ray diffractions, FTIR and TEM, showing that the processes involved are only surface phenomena, not interfering with the bulk structure of the matrices. Only UV-Vis spectra are sensitive enough to probe the existence of the iron porphyrin at the surface of the supports.

In preliminary tests of the oxidation of cyclohexane, all catalyst solids obtained by the immobilization of both iron porphyrins have shown good performance, especially those which were immobilized on silica obtained from acid-leached chrysotile, and catalyst Fe(TDFSPP) being superior to Fe(TCFSPP). Different catalytic results were observed depending on the support, the substituent present in the iron porphyrins and the solvent used during the catalytic activity experiments but the time of the reaction was the factor had that most influence in the heterogeneous catalysts performance.

\section{References}

1. El-Awady, A.A ; Wilkins, P.C.; Wilkins, R.G.; Inorg. Chem. 1985, 24, 2053.

2. Mansuy, D.; Coord. Chem. Rev. 1993, 125, 129.

3. Bedioui, F.; Coord. Chem. Rev. 1995, 144, 39.

4. Haber, J.; Matachowski, L.; Pamin, K.; Poltowicz, J.; J. Mol. Catal. A: Chem. 2003, 198, 215.

5. Benedito, F.L.; Nakagaki, S.; Saczk, A.A.; Peralta-Zamora, P.G.; Costa, M.C.M.; Appl. Catal., A 2003, 250, 1.

6. Iamamoto, Y.; Idemori, Y.M.; Nakagaki, S.; J. Mol. Catal. A: Chem. 1985, 99, 187.

7. Matinez-Lorente, M.A.; Battioni, P.; Kleemiss, W.; Bartoli, J.F.; Mansuy, D.; J. Mol. Catal. A: Chem. 1996, 113, 344.

8. Nakagaki, S.; Xavier, C.R.; Wosniak, A.J.; Mangrich, A.S.; Wypych, F.; Cantão, M.P; Denicoló, I.; Kubota, L.T.; Colloid Surf. A-Physicochem. Eng. Asp. 2000, 168, 261;

9. Herron, N.; Coord. Chem. Rev. 1988, 19, 25.

10. Barloy, L.; Lallier, J. P.; Battioni, P.; Mansuy, D.; Pitfard, Y.; Tournoux, M.; Valim, J. B.; Jones, W.; New J. Chem. 1992, 16, 71.

11. Machado, A. M.; Wypych, F.; Drechsel, S. M.; Nakagaki, S.; J. Colloid Interface Sci. 2002, 254, 158.

12. Cady, S. S.; Pinnavaia, T. J.; Inorg. Chem. 1978, 17, 1501.

13. Chibwe, M.; Ukrainczyk, L.; Boyd, S. A.; Pinnavaia, T. J.; J. Mol. Catal. A: Chem 1996, 113, 249. 
14. Nakagaki, S.; Ramos, A.R.; Benedito, F.L.; Peralta-Zamora, P.G.; Zarbin, A.J.G.; J. Mol. Catal. A: Chem 2002, 185, 203.

15. Nakagaki, S.; Halma, M.; Bail, A.; Arízaga, G. G. C.; Wypych, F.; J. Colloid Interface Sci. 2005, 281, 417.

16. Nakagaki, S.; Benedito, F. L.; Wypych, F.; J. Mol. Catal. A: Chem. 2004, 217, 121.

17. Dolphin, D.; Traylor, T. G.; Xie, L.Y.; Acc. Chem. Res. 1997, $30,251$.

18. Hibino, T.; Jones, W.; J. Mater. Chem. 2001, 11,1321.

19. Halma, M.; Wypych, F.; Dreschel, S. M.; Nakagaki, S.; J. Porphyrins Phthalocyanines 2002, 6, 502.

20. Brindley, G.W.; Brown, G.; Crystal Structure of Clay Minerals and their X-ray Identification, Mineral Society: London, 1980.

21. Wypych, F.; Schreiner, W. H.; Mattoso, N.; Mosca, D. H.; Marangoni R.; Bento, C.A.S.; J. Mater. Chem. 2003, 13, 304.

22. Wypych, F.; Adad, L. B.; Mattoso, N.; Marangon, A. A. S.; Schreiner, W. H.; J. Colloid Interface Sci. 2005, 283, 107.

23. Halma, M.; Bail, A.; Wypych, F.; Nakagaki, S.; J. Mol. Catal. A: Chem. 2006, 243, 44.

24. Wypych, F.; Bail, A.; Halma, M.; Nakagaki, S., J. Catal. 2005, 234, 31.

25. Bonnet, S.; Forano, C.; De Roy, A.; Besse, J. P.; Chem. Mater. 1996, 8, 1962.

26. Sharefkin, J. G.; Saltzmann, H.; Org. Synth. 1963, 43, 62.
27. Lucas, J.; Kennedy, E. R.; Forno, M. W.; Org. Synth. 1963, 43, 483.

28. Lindsey, J. S.; J. Org. Chem. 1987, 54, 827.

29. Rocha-Gonsalves, A. M.; Pereira, M. J.; J. Heterocycl. Chem. 1985, 22, 931.

30. Rocha-Gonsalves, A. M.; Varejão, J. M. T. B.; Pereira, M. M.; J. Heterocycl. Chem. 1991, 28, 635.

31. Smith, K. M.; Porphyrins and Metalloporphyrins, Elsevier: Amsterdam, 1975, p. 317.

32. Adler, A.; Longo, F. R.; J. Am. Chem. Soc. 1964, 86, 3145.

33. Adler, A.; Longo, F. R.; Kampas, F.; Kim, J.; Inorg. Nucl. Chem. 1979, 32, 2443.

34. Wypych, F.; Schreiner, W. H.; Mattoso, N.; Mosca, D. H.; Marangoni, R.; Bento, C. A. S.; J. Mater. Chem. 2003, 13, 304.

35. Fonseca, M. G.; Oliveira, A. S.; Airoldi, C.; J. Colloid Interface Sci. 2001, 240, 533.

36. Kameyama, H.; Suzuki, H.; Amano, A; Chem. Lett. 1988, 1117.

37. Groves, J. T.; Nemo, T. E.; Meyers, R. S.; J. Am. Chem. Soc. 1979, 101, 1032.

38. Groves, J. T.; Haushalter, R. C.; Nakamura, M.; Nemo, T. E.; Evans, B. J.; J. Am. Chem. Soc. 1981, 103, 2884.

39. Fujii, H.; Coord. Chem. Rev. 2002, 226, 51.

Received: June 9, 2006

Published on the web: November 7, 2006 